\title{
Modelagem Matemática e Simulação Computacional de Secadores de Erva-Mate em Leito Fixo
}

B.C. PASA ${ }^{1}$, A. DERIVI ${ }^{2}$, C.A. ZAKRZEVSKI ${ }^{3}$, E.F. ZANOELO ${ }^{4}$, Universidade Regional Integrada do Alto Uruguai e das Missões - URI, Campus de Erechim, Av. Sete de Setembro, 1621, 99700-000 Erechim, RS, Brasil.

Resumo. Neste estudo estimou-se a variação da umidade de erva-mate durante o processo de secagem em leito fixo por modelagem e simulação. Um sistema de equações diferenciais parciais foi proposto através de balanços de massa e energia tomando como referência gás e sólido seco em um elemento de volume da fase. $\mathrm{O}$ modelo matemático foi resolvido através do método das diferenças finitas na direção axial, enquanto que para a integração ao longo do tempo empregou-se o método de Runge-Kutta de ordem 4. Um programa em linguagem computacional FORTRAN foi desenvolvido para implementação dos métodos matemáticos e realização das simulações. Resultados experimentais de umidade de erva-mate ao longo do tempo foram comparados com valores obtidos através da solução do sistema de equações proposto, a fim de validar o modelo matemático. Estimou-se a eficiência energética do processo de secagem de erva-mate em leito fixo para as condições de operação investigadas neste trabalho.

\section{Introdução}

A erva-mate Ilex paraguariensis St. Hil. é uma planta característica do Mercosul pertencente a família Aqüifoliácea. Dentre as 600 espécies que representam esta família, 60 são encontradas no Brasil. A produção brasileira de erva-mate cancheada para preparação de chimarrão ou chá está atualmente em torno de 210 mil toneladas/ano, com receita equivalente a $\mathrm{R} \$ 150$ milhões, conforme [4]. Segundo Rodigheri 1995, [6], a produção nacional está concentrada nos Estados de Santa Catarina (53,6\%), Paraná (23,3\%), Rio Grande do Sul (21,5\%) e Mato Grosso do Sul (1,6\%). O Rio Grande do Sul, que no início da década de 70 era o principal produtor nacional, respondendo por cerca de $50 \%$ da produção do país, teve sua participação diminuída nas últimas décadas, [11].

\footnotetext{
${ }^{1}$ bpasa@bol.com.br

2 aderivi@aol.com

3 caz@uri.com.br

4 everton.zanoelo@uri.com.br
} 
No Alto Uruguai Gaúcho o cultivo e beneficiamento de erva mate é ainda hoje uma atividade geradora de renda de parcela significativa da população do campo e da cidade. Estima-se que 8000 famílias de produtores rurais e aproximadamente 40 indústrias ervateiras dessa região estejam envolvidas na produção de erva-mate cancheada. Os dados atuais somados as recentes projeções de crescimento do setor evidenciam a importância sócio econômica de erva-mate para região do Alto Uruguai, bem como para o estado do Rio Grande do Sul.

Apesar da recente injeção de recursos em pesquisa no setor ervateiro, principalmente destinados a melhoria da qualidade da planta, ainda existe uma grande necessidade de estudos técnicos que tenham como objetivo o desenvolvimento tecnológico do processo de secagem de erva-mate, o qual pode ser considerado como a etapa principal de beneficiamento deste produto. Poucos são os trabalhos científicos cujo objetivo principal, mesmo que de forma indireta, seja otimização do processo de secagem de erva-mate $([5,11])$.

Neste contexto este trabalho visa a modelagem matemática e simulação computacional de secadores de erva-mate em leito fixo para a predição da variação da umidade deste produto ao longo do processo de secagem. A validação do modelo, por comparação entre resultados experimentais e estimados, permite a utilização do mesmo para a predição da eficiência energética do processo nas condições de operação consideradas neste trabalho.

\section{Modelagem Matemática}

As equações necessárias para a simulação do processo de secagem podem ser obtidas a partir de um balanço de massa do soluto e um balanço de energia, para ambas as fases. O sistema de equações proposto pode ser utilizado para a simulação em leitos fixos ou fluidizados desde que os coeficientes de transferência de calor e massa sejam apropriados

$$
\begin{gathered}
G_{g} \frac{\partial Y_{g}}{\partial z}=K_{s} a\left(Y_{s}-Y_{s}^{*}\right) \\
G_{g}\left(C p_{g}+Y_{g} C p_{v}\right) \frac{\partial T_{g}}{\partial z}=-h a\left(T_{g}-T_{s}\right) \\
(1-\epsilon) \rho \frac{\partial Y_{s}}{\partial t}=-K_{s} a\left(Y_{s}-Y_{s}^{*}\right) \\
\rho_{s}(1-\epsilon)\left(C p_{s}+C p_{l} Y_{s}\right) \frac{\partial T_{s}}{\partial t}=h a\left(T_{g}-T_{s}\right)-K_{s} a\left(Y_{s}-Y_{s}^{*}\right)\left[\lambda+C p_{v}\left(T_{g}-T_{s}\right)\right]
\end{gathered}
$$

Pode-se observar inicialmente que a obtenção de perfis de umidade e temperatura do gás e do sólido depende, entre outras propriedades físicas, do conhecimento da 
umidade de equilíbrio $\left(\mathrm{Y}_{s}^{*}\right)$. A umidade de equilíbrio de um material é definida como o teor de umidade deste, correspondente ao equilíbrio entre as pressões de vapor de água no material e no meio ambiente. Mais grosseiramente pode-se dizer que o teor de umidade de equilíbrio é a umidade que o produto atinge quando deixado por tempo suficientemente longo em determinada condição de temperatura e umidade relativa do ar que o envolve. Existem na literatura diferentes equações empíricas para estimativa da umidade de equilíbrio de diversos materiais. Scipioni et al. [7] e, mais recentemente, Menin et al. [5] propuseram correlações empíricas para predição da umidade de equilíbrio de erva-mate Ilex Paraguariensis a diferentes temperaturas e umidades relativas. De acordo com [5], a umidade de equilíbrio pode ser estimada pela equação $(2.5)$

$$
\begin{gathered}
\frac{Y_{s}^{*}}{1+Y_{s}^{*}}=\frac{1}{100}\left[\frac{-e^{\left(-5,7 \times 10^{-3} T_{g}+3,02\right)}}{\ln (U R)}\right]^{\frac{1}{1,51}}, \\
P_{\text {sat }}=e^{\left[18,3036-\left(\frac{3.816,44}{T_{g}+273,15-46,13}\right)\right]} .
\end{gathered}
$$

A umidade relativa pode ser calculada através das equações (2.6) e (2.7), apresentadas por [1]

$$
U R=\left[\frac{692,7}{P_{\mathrm{sat}}}\right]\left[\frac{Y_{g}}{0,625+Y_{g}}\right]
$$

A dependência do calor latente de vaporização da água com a temperatura da superfície da folha é dada pela equação (2.8), segundo [8]. O calor específico da água líquida é aproximadamente constante e igual a $4185,17 \mathrm{~J} / \mathrm{kg}^{\circ} \mathrm{C}$, enquanto o calor específico para a erva-mate foi admitido como sendo igual ao Cp do Chá Verde Japonês, obtido a partir do processamento de Camellia sinensis. De acordo com Yoshitomi [9], o calor específico do Chá Verde Japonês é igual a $960 \mathrm{~J} / \mathrm{kg}^{\circ} \mathrm{C}$. A equação (2.9) permite a estimativa da variação do calor específico do ar e do vapor d'água com a temperatura empregando-se os coeficientes definidos na Tabela 1 ([2])

$$
\begin{gathered}
\lambda=2,255 \times 10^{6}\left[\frac{0,578-1,545 \times 10^{-3} T_{s}}{0,423}\right]^{0,38}, \\
C p=a_{1}+a_{2} T_{g}+a_{3} T_{g}^{2}+a_{4} T_{g}^{3}+a_{5} T_{g}^{4} .
\end{gathered}
$$

A densidade do ar, necessária para a determinação da velocidade mássica, G, é expressa pela equação (2.10). Os coeficientes do polinômio foram ajustados considerando-se o intervalo de temperatura de $-20 \mathrm{a}+180^{\circ} \mathrm{C}$ 
Tabela 1: Coeficientes da equação (2.9) para cálculo do calor específico do ar e vapor d'água [2].

\begin{tabular}{c|c|c}
\hline & $A r$ & Vapor \\
\hline \hline$a_{1}$ & $1.005,6$ & $2.423,23$ \\
\hline$a_{2}$ & $7,31 \times 10^{-3}$ & $-5,564$ \\
\hline$a_{3}$ & $4,32 \times 10^{-4}$ & $2,35 \times 10^{-2}$ \\
\hline$a_{4}$ & 0 & $-3,9 \times 10^{-5}$ \\
\hline$a_{5}$ & 0 & $2,39 \times 10^{-8}$ \\
\hline$T_{g}^{0}$ & $0-300$ & $100-300$ \\
\hline
\end{tabular}

$$
\rho_{g}=1,298-4,584 \times 10^{-3} T_{g}+9,606 \times 10^{-6} T_{g}^{2} .
$$

A eficiência energética do secador é definida de modo simplificado como a razão entre o calor utilizado para evaporação da água contida nas folhas de erva-mate e a energia fornecida para aquecer o ar de secagem, equação (2.11), segundo [3]. Na estimativa da água evaporada durante a secagem considerou-se que a umidade final do produto deveria atingir um valor residual de aproximadamente $8 \%$ (b.s.), sendo ts o tempo transcorrido para que este valor fosse obtido. Desprezou-se neste cálculo a energia mecânica necessária para insuflar o ar quente no leito de secagem, bem como o calor despendido para o aquecimento das folhas

$$
E(\%)=100\left[\frac{m_{l} \lambda}{\dot{m}_{g} C p_{g}\left(T_{g a}-T_{\infty}\right) t s}\right]
$$

Para predição da eficiência energética assumiu-se o calor latente de vaporização constante e igual a $2,256 \times 10^{6} \mathrm{~J} / \mathrm{kg}$. O calor específico do ar foi estimado na média aritmética das temperaturas ambiente e do ar na alimentação. A massa total de água evaporada ao longo do processo de secagem pode ser calculada através da equação (2.12), em função da massa de erva-mate úmida no instante $t=0$ (mso) e da umidade da erva-mate na alimentação em base úmida. A vazão mássica de ar quente é obtida por sua vez através da equação (2.13)

$$
\begin{gathered}
m_{l}=m_{s o}\left[\frac{Y_{s o}}{1+Y_{s o}}\right], \\
\dot{m}_{g}=G_{g}\left[\frac{\pi\left(D_{c}^{2}\right)}{4}\right] .
\end{gathered}
$$




\section{Resultados e Discussões}

O sistema de equações diferenciais parciais definido anteriormente pelas equações (2.1) a (2.4), pode ser resolvido numericamente desde que se conheçam as condições iniciais e de contorno. As primeiras são facilmente obtidas uma vez que no instante inicial, $\mathrm{t}=0$, independente da posição axial no leito, a temperatura e umidade do sólido são conhecidas e definidas respectivamente por Tso e Yso. As condições de contorno são obtidas conhecendo-se a umidade e temperatura do ar quente na alimentação, as quais na posição $\mathrm{z}=0$ independentemente do tempo de secagem transcorrido, são respectivamente Yga e Tga.

Exceto as variáveis estimadas através das equações definidas na etapa de Modelagem Matemática, todas as demais informações requeridas para a simulação do processo de secagem de erva-mate em leito fixo, bem como as respostas obtidas em cada condição de operação, são apresentadas na Tabela 2. Devido a inexistência de expressões empíricas na literatura para a estimativa dos coeficientes de transferência de calor e massa na secagem convectiva de erva-mate, estas variáveis foram obtidas experimentalmente em um secador a leito fixo operando nas condições experimentais definidas na Tabela 2.

Tabela 2: Condições de Operação Variáveis.

\begin{tabular}{c|c|c}
\hline Variaveis & Corrida 1 & Corrida 2 \\
\hline \hline$Y_{g a}$ & $1,66 \times 10^{-3}$ & $4,77 \times 10^{-3}$ \\
\hline$Y_{s o}$ & 1,42 & 1,45 \\
\hline$T_{g a}$ & 51,9 & 47,6 \\
\hline$T_{s o}$ & 18,0 & 18,0 \\
\hline$\rho_{s}$ & 457,45 & 457,45 \\
\hline$h a$ & 346,55 & 470,23 \\
\hline$K_{s} a$ & $2,38 \times 10^{-3}$ & $4,08 \times 10^{-3}$ \\
\hline$\epsilon$ & 0,927 & 0,927 \\
\hline$T_{\infty}$ & 16,5 & 17,5 \\
\hline$U_{g}$ & 0,063 & 0,287 \\
\hline$D_{c}$ & 0,127 & 0,127 \\
\hline$H$ & 0,045 & 0,045 \\
\hline$m_{s o}$ & 0,038 & 0,041 \\
\hline$t s$ & $45.520,0$ & $27.840,0$ \\
\hline$E$ & 3,6 & 1,6 \\
\hline \multicolumn{2}{|c}{}
\end{tabular}

O modelo matemático definido pelas equações (2.1) a (2.4) foi resolvido através do método das diferenças finitas na direção axial, enquanto que para a integração ao longo do tempo empregou-se o método de Runge-Kutta de ordem 4. Um programa em linguagem computacional FORTRAN foi desenvolvido para implementação dos 
métodos e realização das simulações.

Os passos de integração em ambas as direções foram diminuídos ao ponto de não observar-se variação significativa nos perfis de umidade ao longo do tempo. Verificou-se para todas as simulações realizadas que a relação entre os passos na coordenada axial e temporal permitisse a obtenção de uma solução numérica estável.

A fim de verificar a validade do modelo foi feita uma comparação entre perfis temporais de umidade de erva-mate obtidos experimentalmente e preditos através do modelo matemático. Os experimentos requeridos para esta nesta análise foram conduzidos em uma unidade de secagem de bancada, basicamente constituída por um compressor, um elemento de aquecimento de ar, válvulas de regulagem de vazão e um vaso cilíndrico a ser preenchido com as folhas a serem secas. A umidade da erva-mate contida no leito de secagem foi determinada experimentalmente em intervalos regulares de tempo. As condições experimentais de operação do secador são apresentadas na Tabela 2. As Figuras 1 e 2 apresentam um confronto entre resultados experimentais e calculados por solução do modelo apresentado neste trabalho, respectivamente para as condições definidas pelas Corridas 1 e 2 (Tabela 2).

Uma rápida análise das Figuras 1 e 2 demonstra a boa concordância entre os resultados experimentais e calculados, para ambos os casos considerados neste trabalho. A validade do modelo também pode ser verificada através do cálculo dos desvios médios relativos entre resultados preditos e obtidos experimentalmente. Os valores encontrados para estes parâmetros (aproximadamente $5 \%$ e 9\%, respectivamente, para as Figuras 1 e 2) confirmam a consistência do modelo representado pelas equações (2.1) a (2.4).

Convém ressaltar que a solução do modelo matemático permite a obtenção de perfis temporais de umidade para diferentes posições axiais no leito. Entretanto, considerando-se que neste trabalho foram utilizados leitos rasos, não são esperadas variações significativas destes perfis. De fato, resultados obtidos por simulação confirmam este comportamento.

Neste ponto é preciso salientar que umidade de erva-mate decresce com o tempo até atingir um valor limite dado pela umidade de equilíbrio do sólido, segundo as condições de umidade relativa e temperatura. O valor crítico de umidade e o tempo total transcorrido até que este parâmetro fosse atingido não são representados nas Figuras 1 e 2 devido a falta de dados experimentais para comparação com resultados estimados, em tempos superiores a aproximadamente 2 horas. O tempo necessário para que as folhas de erva-mate contidas no leito atingissem uma umidade de $8 \%$ (b.s.) é apresentado na Tabela 2 .

Perfis temporais de temperatura e umidade do gás, para uma dada altura, ou perfis axiais, em um dado instante, podem ser facilmente obtidos por solução do modelo proposto, porém visto que o foco da secagem é a redução de umidade no sólido os resultados representados nas Figuras 1 e 2 dispensam demais apresentações.

Uma observação detalhada da Tabela 2 permite verificar que com exceção da velocidade superficial de ar quente no leito e as variáveis dela dependente, todas as demais condições de operação se mantém aproximadamente constantes para as Corridas 1 e 2. Portanto, torna-se evidente que a diminuição da eficiência energética e do tempo necessário para que a umidade final de $8 \%$ seja atingida é simplesmente 


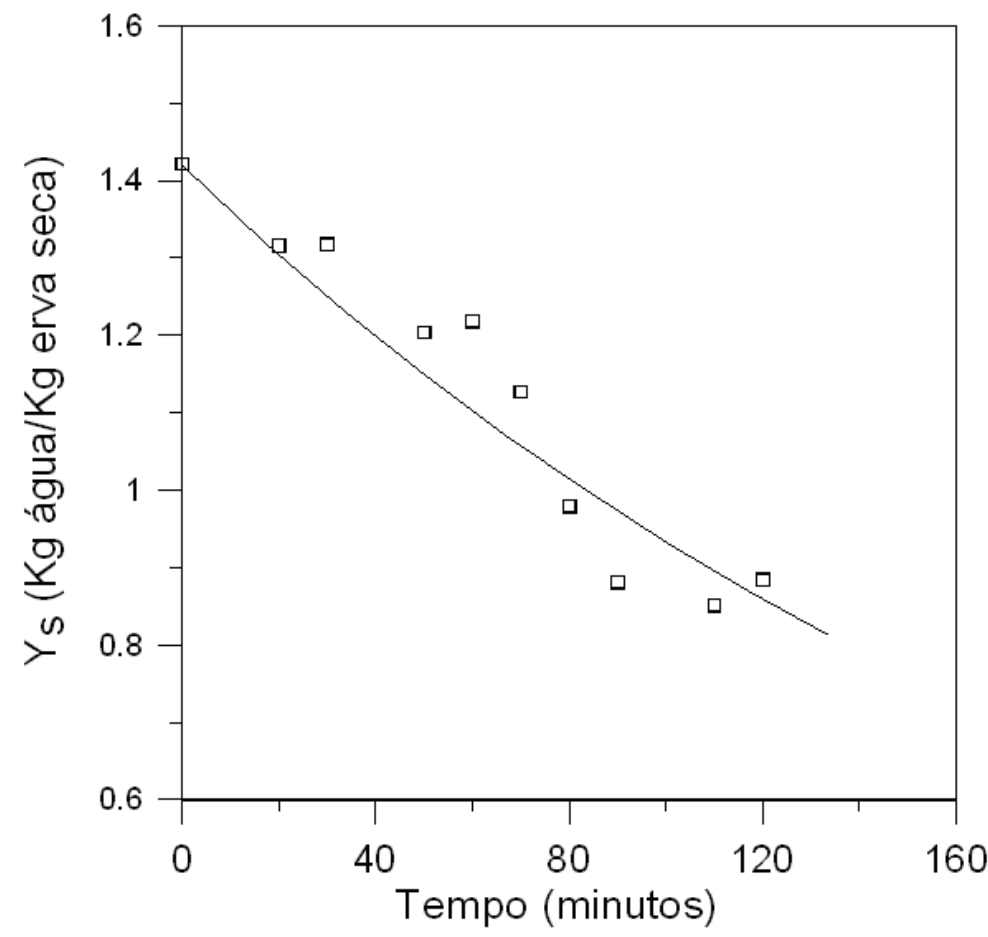

Figura 1. Comparação entre resultados experimentais e calculados para condições de operação definidas na Tabela 2 (Corrida 1).

um efeito do incremento de velocidade no leito.

\section{Conclusões}

Um modelo matemático para a predição da variação da umidade de folhas de erva-mate durante a secagem em leito fixo foi proposto. O sistema de equações diferenciais parciais obtido através de balanços de massa e energia para o soluto em ambas as fases foi resolvido através do método das diferenças finitas na direção axial, enquanto que para a integração na coordenada temporal utilizou-se o método de Runge-Kutta de $4^{a}$ ordem. A comparação entre resultados experimentais e preditos, nas condições de operação investigadas, confirma a validade do modelo. A eficiência energética do secador e o tempo de final de secagem diminuíram com o incremento da velocidade superficial no leito. 


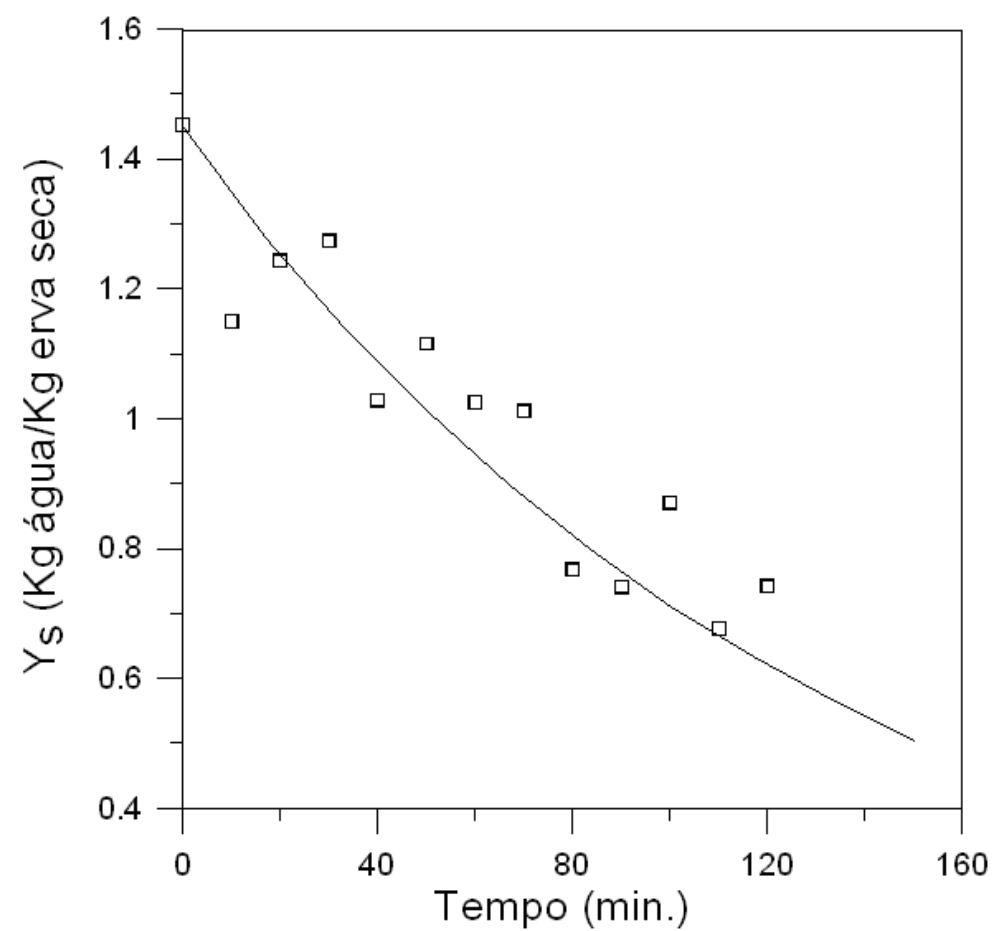

Figura 2. Compar ação entre resultados experimentais e calculados para condições de operação definidas na Tabela 2 (Corrida 2).

\section{Lista de Símbolos}

Cp calor específico à pressão constante, $\frac{\mathrm{J}}{\mathrm{kg}^{\circ} \mathrm{C}}$

E eficiência energética, \%

G velocidade mássica, $\frac{\mathrm{kg}}{\mathrm{m}^{2} \mathrm{~s}}$

ha coeficiente volumétrico efetivo de transferência de calor, $\frac{J}{m^{3} s^{\circ} \mathrm{C}}$

$\mathbf{K}_{\mathbf{s}} \mathbf{a} \quad$ coeficiente volumétrico efetivo de transferência de massa, $\frac{\mathrm{kg}}{\mathrm{m}^{3}}$

m massa, $\mathrm{kg}$

m vazão mássica; $\frac{\mathrm{kg}}{\mathrm{s}}$

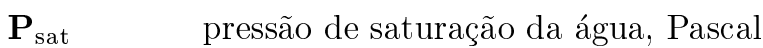

t variável temporal, $\mathrm{s}$

$\mathbf{T}$ temperatura, ${ }^{\circ} \mathrm{C}$

$\boldsymbol{T}_{\infty} \quad$ temperatura ambiente, ${ }^{\circ} \mathrm{C}$

ts tempo de secagem para reduzir a umidade do sólido a $8 \%, \mathrm{~s}$

UR umidade relativa do ar, adimensional

Y fração mássica de água em base seca, $\frac{\mathrm{kg}}{\mathrm{kg}}$ 


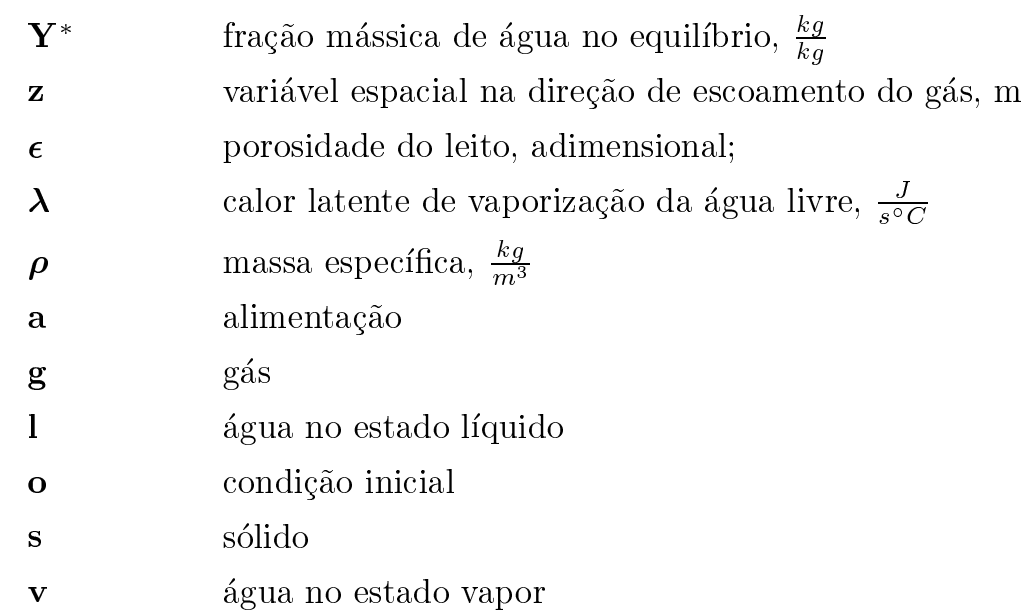

Abstract. In this work the moisture content of maté in a fixed bed dryer was predicted using a mathematical model proposed through mass and energy balances in a volume element of fluid and solid into the bed. The system of partial differential equations was solved numerically by using two different numerical methods. The finite difference method was applied for the spatial integration, while along the time axis was employed the $4^{t h}$ order Runge-Kutta method. A FORTRAN computational program was proposed to simulate the drying process of maté. The experimental and predicted results of moisture content of maté as function of time were compared in order to access the reliability of the mathematical approach. The thermal efficiency of the dryer was calculated for the operating conditions considered in this study.

\section{Referências}

[1] L.A. Calçada, E. Biscaia Jr., e G. Massarani, Modelagem e simulação de secadores em leito fixo, em "Anais do XXI Encontro sobre escoamento em meios porosos", pp.384-395, Ouro Preto/MG, 1993.

[2] J.P. Holman, "Transferência de Calor", McGraw-Hill, (1983).

[3] M.D. Hubinger e F.C. Menegalli, Simulação de Secadores em Leito Deslizante, com Escoamentos Concorrente e Contracorrente. Análise da Eficiência Energética, em "Anais do XXI Encontro sobre escoamento em meios porosos", pp. 522-532, Ouro Preto/MG, 1993.

[4] R.S. Lourenço, M.J. Medrado, J.A.P. Fowler, e S.H. Mosele, Influência do Substrato no Desenvolvimento de Mudas de Erva-Mate, Ilex paraguariensis St. Hil., Revista Perspectiva, 24, No. 88 (2000), 81-99. 
[5] J.C. Menin, B. Pasa, E.F. Zanoelo e J.R. Finzer, Isotermas de Umidade de Equilíbrio para Erva-Mate, em "IV Encontro Brasileiro de Adsorção", Rio de Janeiro, 2002.

[6] H.R. Rodigheri, L. Schlossnacher Neto e I.F. Cichaczewski, "Custos, produtividade e renda da erva-mate cultivada na região de Guarapuava, PR", Colombo: EMBRAPA-CNPF, CT 24, 1995.

[7] G.P. Scipioni, B.E. Meza, S.D. Prat Krikum, B. Del V. Argüello, Comparación de las isotermas de deserción de Ilex dumosa e Ilex paraguarienses St. Hil., em "II Congresso sul-americano da erva-mate, III Reunião Técnica da erva-mate", pp. 359-362, 2000.

[8] J. M. Smith e H.C. Van Ness, "Introdução à Termodinâmica da Engenharia Química", 3 edição, Guanabara Dois, 1980.

[9] H. Yoshitomi, Drying characteristics of tea leaves (III). Effect of primary drying conditions on tea drying, Journal of the Japanese Society of Agricultural Machinery, (1987), 197-206.

[10] A.T. Valduga, J.R.D. Finzer, E.F. Zanoelo e R.L. Krüger, "Secador de Bandeja Vibrada: Transferência de Calor na Secagem de Erva-Mate", em "IX Congresso Brasileiro de Engenharia e Ciências Térmicas", 2002.

[11] E. Valduga, "Caracterização Química e Anatômica da folha de Ilex paraguariensis St. Hill. e de algumas espécies utilizadas na adulteração do Mate", Dissertação de Mestrado, UFPR, 1994. 